\title{
RHEOLOGICAL INVESTIGATIONS OF NON-ISOTHERMAL PRESSURE FLOWS OF COATING MIXTURES FOR WELDING ELECTRODES
}

\author{
A.E. MARCHENKO \\ E.O. Paton Electric Welding Institute, NASU \\ 11 Kazimir Malevich Str., 03680, Kiev, Ukraine. E-mail: office@paton.kiev.ua
}

\begin{abstract}
Continuous flow capillary viscosimeter, presented in the form of abruptly narrowing stepped channel, and INSTRON 1251 testing machine were used to model the processes of pressure flow of coating mixtures for welding electrodes in extrusion chamber and in die space of production extruding machine. Thermocouple measurements were used to study the thermal conditions in pre-capillary zone, and visualization method was applied to analyze the structure of flow in it. Mathematical apparatus developed for calculation of rheological characteristics of polymer materials in the state of viscoelastic pressure flow was applied to calculate similar characteristics of the two types of coating mixtures for two types of low-hydrogen electrodes made with application of viscous in one and low-viscous liquid glass in another one. Grain composition of their filler is the same. Values of viscoelastic characteristics of the mixtures were calculated, including shear and longitudinal viscosity, elastic potential, first difference and coefficient of first difference of normal stresses and the relaxation period. Their dependencies on geometrical dimensions of stepped channels and values of volume flow of the mixture at capillary measurements were analyzed and commented upon, allowing for suspension nature of the mixtures, as well as resistance of pressure flows formed in them to viscoelastic instability. 12 Ref., 3 Tables, 12 Figures.
\end{abstract}

Keywords: rheology, welding electrodes, mixtures characteristics of mixtures, pressure flow instabilities

As the coating mixture passes from the working cylinder of extruding machine into the sizing die, in which the coating mixture envelops the metal rods, its flow cross-section decreases, and its rate rises by tens of times. Here, the mixture properties, which determine the manufacturing properties and working characteristics of welding electrodes, change essentially. For many years it was not known exactly which of these properties change, to what extent and in which way. Essentially, this is why the very physicochemical nature of coating mixtures proper remained unclear. The work [1] published 65 years ago, even though it did not disclose this nature, nonetheless, provided objective evidence of the fact that this cannot be done, proceeding just from the concepts of electrode coating mixture viscosity.

Application of colloidal materials science and engineering rheology approaches at PWI for the last 10-15 years allowed establishing that electrode coating mixtures, similar to other kinds of highlyconcentrated suspensions of hard particles in viscous liquid medium, are characterized by elastic properties alongside viscosity. Not only shear and longitudinal viscosity, but a number of elasticity characteristics should be used for complete description of their rheological properties.
The elastic component, first of all, essentially improves energy consumption for organizing the mixture pressure flow for their application on electrode rods. Alongside that, certain combinations of mixture viscosity and elasticity cause their unstable flow. At pressing of welding electrodes, it results in different thickness of the coating, markedly impairing the electrode technological properties. The above instability, as a rule, develops at the transition stage of flow formation, while it, passing through the forming channels in the extruding machine or in the measuring cell of the capillary viscosimeter, after change of its section, direction or rate, reaches the steady-state flow mode. It can develop both in the capillary, and in the zone before it [2-4].

Experimental study of the causes for or mathematical modeling of the conditions, provoking appearance of instabilities of coating mixture pressure flows, should be performed, as in polymer rheology, with application of stepped channel model of continuous-flow type in the first case. In it the flow section decreases abruptly (step-like) or more seldom, gradually narrowing, at transition from the tank (mixture container) to the die (or capillary). Here, the flow is accelerated, either by increasing the degree of jet reduction at unchanged tempo of mixture injection into the die, or by increasing the plunger speed at unchanged degree of reduction. 
The paper presents the results of PWI investigations of pressure flow of electrode coating mixtures through round continuous-flow stepped channels. The objective of investigations is to clarify the extent of the influence of channel geometry and techniques of regulation of flow modes on the temperature, structure, rheological parameters and of stability characteristics of electrode coating mixture pressure flows.

\section{Methods and objects of investigation.} Rheological properties of electrode coating mixtures were studied, mainly, with application of capillary viscosimeterOB 1435 - continuous flow extruder with electromechanical drive. Flow quantity is regulated in steps by replacement of gears and capillaries [5]. In factory model of viscosimeter at diameter of standard working cylinder (tank) $D_{R}=30 \mathrm{~mm}$ the flow is regulated in the range from 1 to $25 \mathrm{~cm}^{3} \cdot \mathrm{s}^{-1}$. Using a capillary with diameter $d_{c}=2-6 \mathrm{~mm}$, average gradient of shear rate can be assigned in the range from 1 to $8 \cdot 10^{3} \mathrm{~s}^{-1}$. It was insufficient to achieve the objectives of planned research. To expand the range of shear rate gradients $\dot{\gamma}_{\mathrm{sh}}$ towards creep flow modes $\left(Q<1 \mathrm{~cm}^{3} \cdot \mathrm{s}^{-1}, \dot{\gamma}_{\mathrm{sh}}\right.$ up to $\left.0.1 \mathrm{~s}^{-1}\right)$, tanks of 15 and $10 \mathrm{~mm}$ diameter were used alongside the standard one. Performed rheological measurements included both the entrance and capillary zone. Extruding the mixture through holes of 2, 4 and $6 \mathrm{~mm}$ diameter in a flat disc $1 \mathrm{~mm}$ thick, entrance zone resistance $P_{0}$ was measured. Capillaries of reduced length $L=10 d_{\text {c }}$ were applied to assess the total pressure losses in measuring cell, including the entrance zone and capillary. Values of $P_{0}$ and $\left(P_{L}-P_{0}\right)$ were used to calculate the required rheological parameters of mixtures characterizing their technological properties, namely:

- shear $\tau$, longitudinal $\sigma$ stresses, as well as the first difference of normal stresses $\sigma_{1}$;

- shear $\lambda$ and longitudinal $\eta$ viscosities generated by these stresses and normal stress coefficient $\xi$;

- angle of natural convergence $\alpha_{0}$;

- relaxation period of elastic stresses $\theta$;

- value of first difference of normal stresses $\sigma_{1} / \tau$ normalized by $\tau$.

Shear rate gradient and stress were calculated by the formulas $\dot{\gamma}_{\mathrm{sh}}=8 Q / \pi d_{\mathrm{c}}^{3}$ and $\tau=\left(P_{L}-P_{0}\right) d_{\mathrm{c}} / 4 L$. Above-mentioned procedures of measurement and calculation, as well as other rheological indices used in this study, are described in detail in $[2,4,5]$.

Table 1 gives the complete plan and results of experiments.

Non-standard tanks of 50, 70 and $90 \mathrm{~mm}$ diameter were used to expand $\dot{\gamma}_{\text {sh }}$ range towards greater values, so that maximum possible value of mixture flow per second $Q$ could reach $200 \mathrm{~cm}^{3} \cdot \mathrm{s}^{-1}$. INSTRON 1251 testing machine was used to provide test loads, sufficient for mixture extrusion from tanks of such a large cross-section. In this case, rheological measurements were limited by entrance zone, i.e. by determination of just $P_{0}$ parameter.

Thermal conditions in the flow zone were studied with application of chromel-alumel thermocouple, measuring junction of which was placed into a circular recess at capillary output edge $L=3 d_{\mathrm{c}}$,

Table 1. Plan and results of experiments used for calculation of rheological characteristics of S2 and N2 coating mixtures

\begin{tabular}{|c|c|c|c|c|c|c|c|c|c|c|c|c|}
\hline \multirow{2}{*}{$d_{c}, \mathrm{~mm}$} & $D_{R} / d_{\mathrm{c}}$ & $Q, \mathrm{~cm}^{3} \cdot \mathrm{s}^{-1}$ & $P_{0}, \mathrm{MPa}$ & $P_{L}, \mathrm{MPa}$ & $D_{R} / d_{\mathrm{c}}$ & $Q, \mathrm{~cm}^{3} \cdot \mathrm{s}^{-1}$ & $P_{0}, \mathrm{MPa}$ & $P_{L}, \mathrm{MPa}$ & $D_{R} / d_{c}$ & $Q, \mathrm{~cm}^{3} \cdot \mathrm{s}^{-1}$ & $P_{0}, \mathrm{MPa}$ & $P_{L}, \mathrm{MPa}$ \\
\hline & \multicolumn{4}{|c|}{$D_{R}=10 \mathrm{~mm}$} & \multicolumn{4}{|c|}{$D_{R}=15 \mathrm{~mm}$} & \multicolumn{4}{|c|}{$D_{R}=30 \mathrm{~mm}$} \\
\hline \multicolumn{13}{|c|}{ Mixture S2 } \\
\hline \multirow{3}{*}{2} & \multirow{3}{*}{5.0} & 0.11 & 18 & 45 & \multirow{3}{*}{7.5} & 0.25 & 24 & 46 & \multirow{3}{*}{15.0} & 1.0 & 10 & 30 \\
\hline & & 0.57 & 18 & 63 & & 1.28 & 26 & 50 & & 5.1 & 15 & 40 \\
\hline & & 1.70 & 27 & 72 & & 3.82 & 12 & 52 & & 15.3 & 18 & 54 \\
\hline \multirow{3}{*}{4} & \multirow{3}{*}{2.5} & 0.11 & 27 & 72 & \multirow{3}{*}{3.75} & 0.25 & 12 & 32 & \multirow{3}{*}{7.5} & 1.0 & 8 & 22 \\
\hline & & 0.57 & 27 & 90 & & 1.28 & 16 & 32 & & 5.1 & 10 & 34 \\
\hline & & 1.70 & 45 & 99 & & 3.82 & 20 & 40 & & 15.3 & 11 & 44 \\
\hline \multirow{3}{*}{6} & \multirow{3}{*}{1.7} & 0.11 & 18 & 56 & \multirow{3}{*}{2.5} & 0.25 & 8 & 36 & \multirow{3}{*}{5.0} & 1.0 & 6 & 22 \\
\hline & & 0.57 & 18 & 59 & & 1.28 & 12 & 36 & & 5.1 & 8 & 22 \\
\hline & & 1.70 & 36 & 72 & & 3.82 & 12 & 36 & & 15.3 & 10 & 32 \\
\hline \multicolumn{13}{|c|}{ Mixture N2 } \\
\hline \multirow{3}{*}{2} & \multirow{3}{*}{5.0} & 0.11 & 16 & 31.5 & \multirow{3}{*}{7.5} & 0.25 & 24 & 32 & \multirow{3}{*}{15.0} & 1.0 & 16.5 & 26 \\
\hline & & 0.57 & 16 & 31.8 & & 1.28 & 20 & 36 & & 5.1 & 16.5 & 35 \\
\hline & & 1.70 & 16 & 45 & & 3.82 & 16 & 46 & & 15.3 & 19.5 & 41 \\
\hline \multirow{3}{*}{4} & \multirow{3}{*}{2.5} & 0.11 & 18 & 36 & \multirow{3}{*}{3.75} & 0.25 & 20 & 32 & \multirow{3}{*}{7.5} & 1.0 & 14.5 & 28.5 \\
\hline & & 0.57 & 10 & 27 & & 1.28 & 16 & 32 & & 5.1 & 14.5 & 44.5 \\
\hline & & 1.70 & 13.5 & 36 & & 3.82 & 14 & 36 & & 15.3 & 14.5 & 28.5 \\
\hline \multirow{3}{*}{6} & \multirow{3}{*}{1.7} & 0.11 & 14.5 & 54 & \multirow{3}{*}{2.5} & 0.25 & 12 & 40 & \multirow{3}{*}{5.0} & 1.0 & 13.5 & 27 \\
\hline & & 0.57 & 14.5 & 37.8 & & 1.28 & 12 & 38 & & 5.1 & 13.5 & 32 \\
\hline & & 1.70 & 9 & 22.5 & & 3.82 & 12 & 32 & & 15.3 & 9.5 & 37 \\
\hline
\end{tabular}

Note. Grey colour denotes anomalous cases characteristic for structured mixtures, bold symbols denote experiments on flow structure visualization. 
Table 2. Composition and characteristics of $\mathrm{NaK}$ liquid glasses

\begin{tabular}{|c|c|c|c|c|c|}
\hline \multirow{2}{*}{$\begin{array}{l}\text { Mixture } \\
\text { index }\end{array}$} & \multicolumn{2}{|c|}{ Liquid glass characteristic } & \multirow{2}{*}{$\begin{array}{l}\text { Fraction of liquid } \\
\text { glass/water, wt.\% }\end{array}$} & \multirow{2}{*}{$\begin{array}{l}\text { Mixture } P_{m}, \\
\quad \mathrm{MPa}\end{array}$} & \multirow[b]{2}{*}{ Instrument name; container diameter $D_{R}$, mm } \\
\hline & Modulus & $\begin{array}{c}\text { Viscosity, } \\
\mathrm{mPa} \cdot \mathrm{s}\end{array}$ & & & \\
\hline S2 & 2.95 & 500 & $27.0 / 0$ & 0.10 & \multirow{2}{*}{ Capillary viscosimeter OB $1435 ; 10,15$ and 30} \\
\hline $\mathrm{N} 2$ & 3.15 & 80 & $14.5 / 4.5$ & 0.75 & \\
\hline S3 & 2.90 & 1000 & $26.5 / 0$ & 0.10 & \multirow{2}{*}{ INSTRON testing machine; 50, 70 and 90} \\
\hline N3 & 3.30 & 100 & $24.0 / 0$ & 0.60 & \\
\hline
\end{tabular}

pressing it tightly to the case by a rubber ring. Temperature was controlled in each experiment. If required, temperature was recalculated to capillaries with $L=10 d_{c}$, used for measurement of rheological indices, by the values of dissipative function $\tau V$ [6]. Temperature of thermocouple cold end was equal to $25{ }^{\circ} \mathrm{C}$.

For visualization of mixture flow profile in the entrance zone, the method of mixture painting with phenolphtalein was used. Experiments were performed in OB 1435 viscosimeter. The billet composed of alternating discs was pre-pressed from painted and unpainted mixtures, was extruded from the respective mixture tank of the viscosimeter through a flat disc with hole diameter of $4 \mathrm{~mm}$ at minimum and maximum plunger feed rate $V_{R}$, equal to 0.15 and $2.15 \mathrm{~cm} \cdot \mathrm{min}^{-1}$. Shear rate gradient in the hole here was equal to 40 and $610\left(D_{R}=30 \mathrm{~mm}\right), 0.25$ and $3.80\left(D_{R}=15 \mathrm{~mm}\right)$, as well as 0.10 and $1.70 \mathrm{~s}^{-1}$ $\left(D_{R}=10 \mathrm{~mm}\right)$. In Table 1 the results of $P_{0}$ and $P_{L}$ measurements taken in these modes are shown in bold. At the right moment the rod was stopped. The rest of the briquette was taken out of the tank, cut along the meridian plane and photographed.

Experimental coating mixtures with filler material composition similar to that of UONI $13 / 55$ electrode coating were studied. In the dry mixture composition,

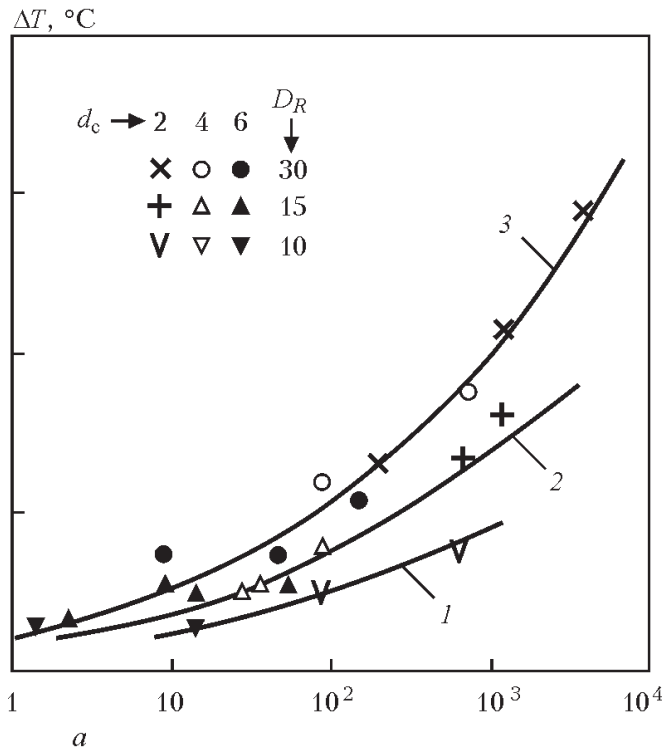

the fraction of particles smaller than $0.063 \mathrm{~mm}$ was equal to $30 \%$, and fraction ratio is close to the one, which provides minimum share of intergranular voids in the compacted layer. $\mathrm{NaK}$ was used as liquid glass to prepare the coating mixtures. Table 2 gives its characteristics and application conditions. In S2 and S3 mixtures made with glasses with viscosity of 500 and $1000 \mathrm{mPa} \cdot \mathrm{s}$, the viscous component of pressure losses prevails over the elastic one. Owing to low viscosity of the binder, N2 and N3 mixtures are characterized by a sufficient degree of structuredness and, therefore, relatively high elasticity. This is indicated by their high plastic strength $P_{m}$. Mixtures, indexed as S2 and N2, were studied with application of OB 1435 viscosimeter, and S3 and N3 mixtures in INSTRON testing machine.

$1 \%$ of powder-like Na-KMTs of $85 \mathrm{SO}$ grade was added to coatings with $\mathrm{S}$ index, and $1.5 \%$ of it was added to those with $\mathrm{N}$ index. Coating plastic strength $P_{m}$ was determined by conical plastometer. Coating mixtures were prepared in laboratory intensive mixer.

Test results. Thermal conditions in the flow zone can be judged by the results of measurements given in Figure 1. They show that N2 mixture in the condition of pressure flow, formed in stepped channels with different tank sections, is heated to lower temperature than S2 mixture, in view of its higher structuredness.

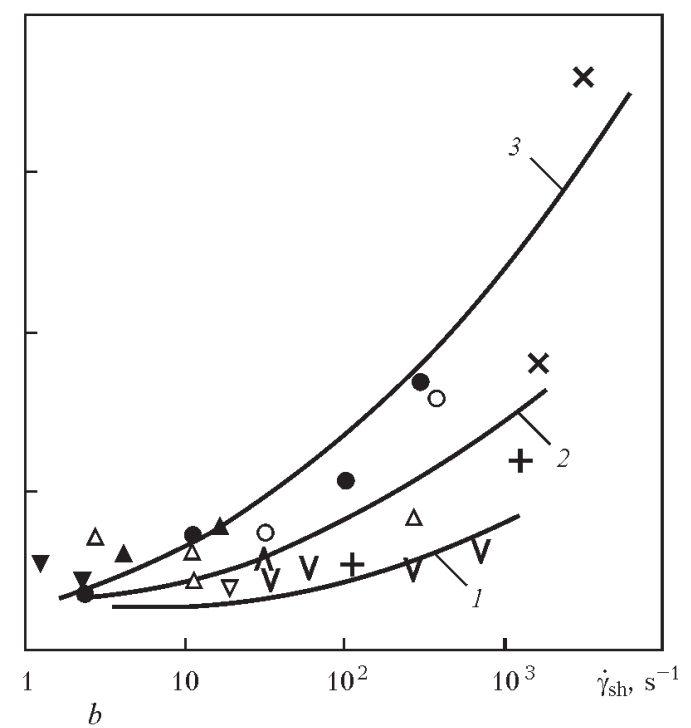

Figure 1. Change of temperature of S2 $(a)$ and N2 $(b)$ coating mixture jet as a result of their viscous heating, depending on average gradient of shear rate at $D_{R}=10(1), 15$ (2) and 30 (3) $\mathrm{mm}$ and $d_{\mathrm{c}}=2,4$ and $6 \mathrm{~mm}$ 
Maximum temperature of the flow of both the mixtures rose progressively with $\dot{\gamma}$ increase and reached $55-65{ }^{\circ} \mathrm{C}$, respectively. It decreased with reduction of tank diameter the greater, the higher $\dot{\gamma}_{\text {sh }}$. As follows from the above graphs, $\Delta t=f\left(\dot{\gamma}_{\mathrm{sh}}\right)$ dependencies were stratified in three levels. Located above all the others are the results obtained at $D_{R}=$ $=30 \mathrm{~mm}$ (curve 3 ), in the middle are the results found at $D_{R}=15 \mathrm{~mm}$ (curve 2), and the lowest ones are those obtained at $D_{R}=10 \mathrm{~mm}$ (curve 1). Jet temperature rises under the influence of $Q$ flow increase and decreases with increase of the degree of jet reduction $\beta=D_{R} / d_{c}$. At $\beta=$ const and $V_{R}=$ const jet temperature was higher, when larger section tank was used, and the more so, the higher $V_{R}$. This is related to increase of the flow, greater in the second case than in the first one.

Similar regularity was observed also in our previous research, in which the jet temperature was recorded at $D_{R}=30 \mathrm{~mm}$, changing just the flow and diameter of the capillary [5].

A significant influence of jet heating on mixture viscosity can be anticipated in experiments with $D_{R}=$ $=30 \mathrm{~mm}$, conducted at maximum flow per second. Temperature of the jet flowing out of the nozzle in creep flow mode $\left(D_{R}=10 \mathrm{~mm}\right)$ cannot influence the coating viscosity, even if capillaries with $L=10 d_{\text {c }}$ are used.

Flow profile. Results of visualization of patterns of S2 and N2 mixture pressure flow are shown in Figure 2. The given photos reflect the flow condition

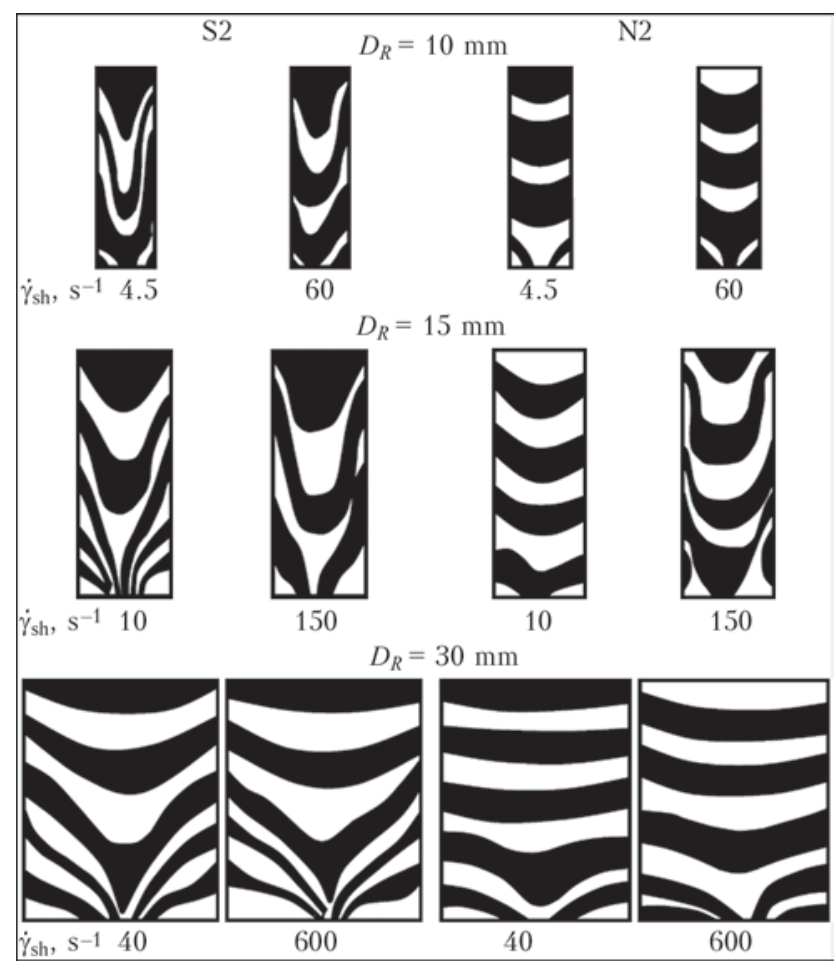

Figure 2. Flow profile of S2 and N2 mixture in the tank of capillary viscosimeter with $D_{R}=10,15$ and $30 \mathrm{~mm}$ through hole of 4 mm diameter at the moment of rod stopping. They demonstrate that under the conditions of laminar flow, the mixture flows by the mechanism of mutual telescopic displacement of the layers, which is complicated by large, nonuniform and non-monotonic deformations. Judging by the shape of flow lines in the entrance zone, flow structures of the compared mixtures, differing essentially by the degree of structuredness, react differently to change of outflow rate and diameter of the tank.

Let us first consider the flow of S2 mixture with viscous liquid glass in the container of $D_{R}=10 \mathrm{~mm}$ at minimum flow $\left(\dot{\gamma}_{\text {sh }}=4.5 \mathrm{~s}^{-1}\right)$. Here slow (creep) flow is in place. Long time before coming to outlet hole the central longitudinal layers of the billet, the least affected by the braking action of the tank cylindrical wall, are significantly accelerated, and, moving by the shear mechanism, form, together with other layers, a flow profile close to the parabolic one, as in an infinite pipe. The profile is almost flat in its core, with the same thickness as that of the initial layer. On the boundary with the tank wall, the layer thickness is minimum. With increase of the flow up to the maximum one $\left(60 \mathrm{~s}^{-1}\right)$ there are indications of coating sliding along the tank wall, so that the initial layer thickness on the wall is preserved for a longer time at this rate.

In the tank of $15 \mathrm{~mm}$ diameter $\left(\dot{\gamma}_{\mathrm{sh}}=10 \mathrm{~s}^{-1}\right)$ dissipative heating of the coating begins to affect its flow, and its pattern becomes more complicated. Layer thickness decreases closer to the outlet hole. This should be promoted also by «pressing» action of stagnation zones, created in the solid angle, formed by the tank cylindrical and end walls. This is confirmed by the horn shape of the flow closer to the outlet hole. In the experiment with $\dot{\gamma}_{\mathrm{sh}}=150 \mathrm{~s}^{-1}$ a cohesion stalling of the flow is observed, which can be regarded as the result of discharging of elastic energy, accumulated in the mixture in the previous deformation period. Indications of pressing action of elastic stresses were more clearly manifested here than in other experiments. A very narrow convergent angle at the entrance to the hole most probably forms under their influence. A similar flow pattern was observed in the pressure flow of plasticine, used to simulate the pattern of metal billet flow in work [7]. The deformation zone still is of quite considerable extent.

At container diameter of $30 \mathrm{~mm}$, the flow, irrespective of its rate, acquires the horn shape almost immediately after its movement starts, and the space, in which deformation occurs, still takes a quite large volume.

N2 mixture in narrow containers demonstrates the cork profile of the flow. Compared to S2 mixture, 
its deformation is concentrated in the short zone, directly adjacent to outlet nozzle. Apparently, under the influence of accumulated elastic stresses, the magnitude of which for this type of mixture is anticipated to be higher than that of S2 mixture, at container diameter of $15 \mathrm{~mm}$ and $\dot{\gamma}_{\mathrm{sh}}=150 \mathrm{~s}^{-1}$ signs of its sticking to container side wall appear (deformed volume of the mixture rises significantly at the same time). In this case, an obvious jet stalling is recorded, resulting from relieving of elastic stresses, which, apparently, is accompanied by its heating. When breaking through to the outlet hole, the flow overcame the pressing action of the stagnation zone. The photo shows its circular residue, adjacent to container edge surface.

In the container of $30 \mathrm{~mm}$ diameter, the deformation zone with weakly pronounced horn-shaped flow profile, directly adjacent to the outlet hole, was again reduced in volume.

In all the photos, indications of flow non-monotonic nature are also visible, alongside the above-described manifestations of its non-uniformity. The former, in our opinion, are manifested, primarily, as deviations of deformed layers profile from the axially symmetric shape. It is important to note that flow profile formation in viscous mixture starts a long way before the capillary mouth, which the mixture enters with an already formed profile. In low-viscosity mixture this process just begins on approach to the capillary. It is completed, apparently, in the capillary initial section. This is exactly where pulsation of the coating mixture jet [2] and of polymer melts [8] begins.

Reaction of low-viscosity mixture flow to capillary elongation is also unusual that is described below.

Extrusion curves. Similar to experiments, conducted with application of capillary viscosimeter with working cylinder with $D_{R}=30 \mathrm{~mm}$, the results of which are published in [2-4], it was found that the nature of extrusion curves depends on the consistency of coating mixtures and their pressure flow modes. In S2 mixtures with viscous liquid-glass binder, all of them, irrespective of the container section, have a smoothed shape, which is little affected by the flow rate. This means the tempo of pressure rise at initial deformation stage, its surge at the moment of flow beginning, and tempo of subsequent lowering, right up to reaching the steady-state stage. Increase of deformation rate smoothes all the starting peaks, as accumulation of elastic stresses in the flow is essentially accelerated [2]. In the experiments with working cylinder with $D_{R}=15 \mathrm{~mm}$ and $d_{\mathrm{c}}=4 \mathrm{~mm}$, curves of very interesting shape were observed, characterized by slow pressure build-up and its lowering after achievement of peak value (Figure 3). Such a shape
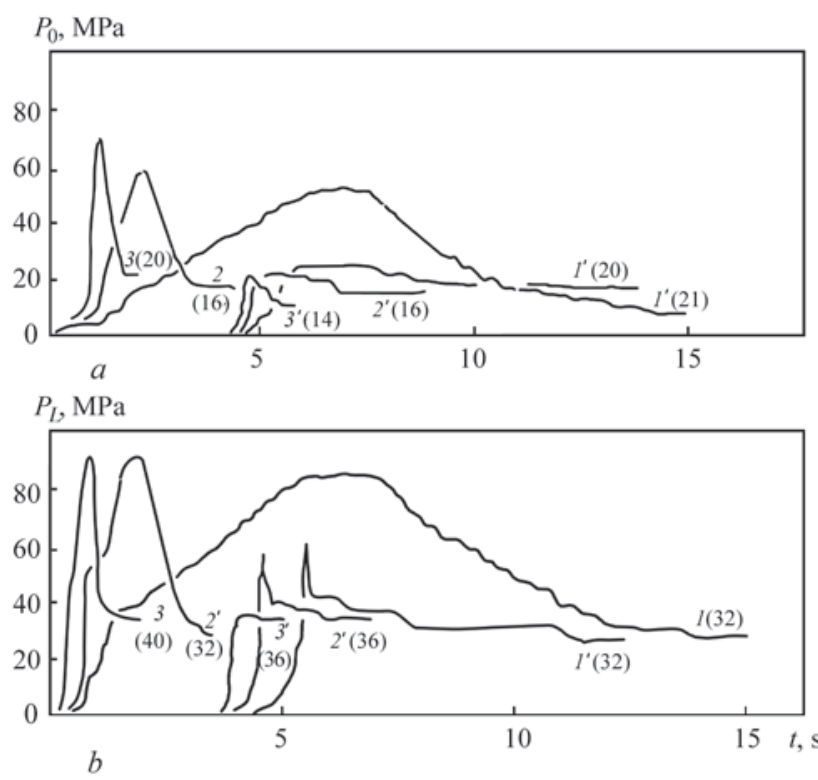

Figure 3. Shape of extrusion curves $P_{0}=f(t)(a)$ and $P_{L}=f(t)(b)$ recorded at testing coating mixtures $\mathrm{S} 2(1,2,3)$ and $\mathrm{N} 2\left(1^{\prime}, 2^{\prime}\right.$, $\left.3^{\prime}\right)$ at shear rate gradient of 10,50 and $150 \mathrm{~s}^{-1}$, respectively, at $D_{R}=15 \mathrm{~mm}$ and $d_{\mathrm{c}}=4 \mathrm{~mm}$ (pressure values are given in brackets) is indicative of low and approximately equal rates of elastic stress accumulation and relaxation that is characteristic for completion of the creep flow stage. It was exactly in this experiment that visualization revealed the profiles, characteristic of flow stalling. In the extrusion curves they are manifested in the form of falling of the descending branch of pressure evolution below the level, recorded in the previous, less fast flow mode. As follows from the data, given in Table 1, this kind of stalling in viscous S2 mixture was recorded in this experiment only for curve $P_{0}=f(t)$. This is also confirmed by its shape in Figure 3. In $P_{L}=f(t)$ curve no elastic pressure relief was observed. This leads to the conclusion that the capillary inner surface in the absence of near-wall slipping is capable of containing elastic stress relaxation up to a certain moment.

In N2 coating mixture, made with low-viscous liquid glass, the tempo of rising, as well as lowering of peak pressure is, as a rule, essentially higher, particularly, when it concerns $P_{L}=f(t)$ curves. Judging by the number of shaded cells in Table 1, there are many more such anomalous cases, characteristic for structured mixtures [2-4], than in its viscous analog under the same flow conditions. In two experiments out of six, it was found for $P_{0}=f(t)$ and $P_{L}=f(t)$, in three experiments - just for $P_{0}=f(t)$, and in two cases just for $P_{L}=f(t)$. This leads to the conclusion that the capillary surface is capable of not only delaying, but under certain conditions provoking an instantaneous relaxation of elastic stresses, for instance, under the influence of dissipative heating, as well as a result of near-wall slipping of the jet by the adhesion, or, less frequently, by the cohesion mechanism [8]. 
Such pressure releases are one of the indications of flow stability disturbance under the influence of elastic stress relaxation.

Flow curves. $P_{0}=f\left(\dot{\gamma}_{\mathrm{sh}}\right)$ and $\tau=f\left(\dot{\gamma}_{\mathrm{sh}}\right)$ flow curves are presented in the graphic form results of simultaneous analysis of visualized patterns and synchronously recorded mode parameters of pressure flow of coating mixtures. In compared mixture flows through stepped channels they, similar to other viscoelastic materials, have the form of curves in logarithmic homology, inclined to rate gradient axis to a larger or smaller degree. Flow curves of S2 mixture are characterized by a larger angle of inclination than in N2 mixture, as the first is closer as to its properties to the Newtonian fluid than is the second one.

In such a systematized and convenient for consideration form, they, in our opinion, provide documented confirmation of the following important points.

The structure and rheological parameters of pressure flow of coating mixtures in stepped channel form under the influence of disturbing action of the tank edge wall, abrupt reduction of the jet at the entrance to the capillary, adhesion braking of the flow by tank and capillary walls, its viscous heating in the zone of maximum shear deformation, accumulation and relaxation of elastic stresses. Some of the above
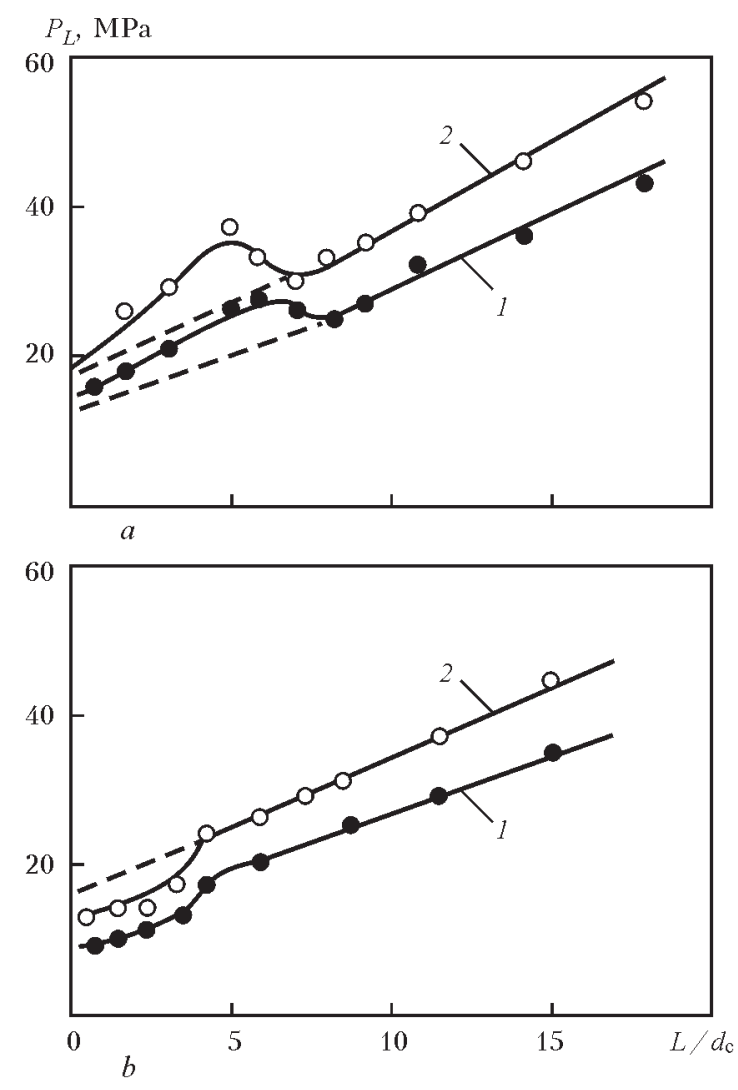

Figure 4. Dependence of total pressure gradient on channel $L / d$ ratio at low-viscosity mixture flow rates of 1 (1) and $15.3 \mathrm{~cm}^{3} \cdot \mathrm{s}^{-1}$ (2) atnd $d_{\mathrm{c}}=2(a)$ and $6(b) \mathrm{mm}$ factors influence the mixture indices adiabatically relative to each other. Mixture flows react to the above-mentioned kinds of disturbances by changing the balance of energy consumption for flow both in the tank, and in the capillary. For instance, if viscosity of S2 and N2 mixtures prevails over elasticity, and this is in place at $D_{R}=30 \mathrm{~mm}$, their flow curves $P_{0}=$ $=f\left(\dot{\gamma}_{\mathrm{sh}}\right)$ are invariant, i.e. independent on volume flow and capillary section. In experiments with smaller containers, figurative points move down as a result of elastic stress relaxation to more favourable energy levels of the flow.

Results, obtained in experiments with smaller diameter tanks, were stratified due to downward individual or group displacements of figurative points, reflecting lowering of flow resistance. Initial invariant flow curve is here stratified into two or three straight lines (by the number of capillaries used).

So, at $D_{R}=15 \mathrm{~mm}$ one result, which was obtained at $d_{\mathrm{c}}=2 \mathrm{~mm}$, shifted from invariant curve $P_{0}=f\left(\dot{\gamma}_{\mathrm{sh}}\right)$; at $D_{R}=10 \mathrm{~mm}$ the results were stratified by three levels, appearing on three mutually parallel lines. Points reflecting the results obtained at $d_{\mathrm{c}}=2 \mathrm{~mm}$ shifted to the lowest curve. Two results obtained at $d_{c}=4 \mathrm{~mm}$ moved to the middle curve (third one was obtained at $d_{\mathrm{c}}=6 \mathrm{~mm}$ ); two results obtained at $d_{\mathrm{c}}=6 \mathrm{~mm}$, appeared on the upper curve (third one was obtained at $d_{\mathrm{c}}=4 \mathrm{~mm}$ ).

As was noted above, stratification of $P_{0}=f\left(\dot{\gamma}_{\mathrm{sh}}\right)$ flow curves is caused by relaxation pressure relieving and viscous heating of the jet, recorded on descending branches of the respective extrusion curves. Parallel displacement of flow curve logarithmic monologues is indicative of unchanged flow structure.

In low-viscosity N2 mixture the initial $P_{0}=f\left(\dot{\gamma}_{\text {sh }}\right)$ flow curve was also stratified but now not only by height, but also by inclination to the axis of shear rate gradients, particularly at $D_{R}=15 \mathrm{~mm}$, that is indicative of the change of not only energy state of the system, but also of flow structure.

The path of $\tau=f\left(\dot{\gamma}_{\text {sh }}\right)$ flow curves of S2 and N2 mixtures is even more complicated. This is related to a greater number of disturbing factors, the action of which depends not only on mode parameters, but also on the structure of mixtures being compared. For instance, in more structured N2 mixture, dissipative heating is less pronounced, but effects of near-wall slipping are more intensively manifested. It can be assumed that the stage of hydrodynamic, temperature and relaxation stationarity of the flow, not completed in pre-capillary zone of the measuring cell, shifts to the initial section of capillary cannel.

As follows from Figure 4, the total pressure gradient at gradual increase of reduced capillary 
length for low-viscosity mixture increases nonmonotonically. At achievement of certain $L / d_{c}$ value, relieving of accumulated elastic stress takes place, and rectilinear branch of $P_{L}=f\left(L / d_{c}\right)$ curve begins only after that. The smaller the capillary diameter, and the larger the mixture flow per second, the greater the pressure relief after the maximum has been achieved. No such anomaly is found in large-diameter capillaries. At passing of high-viscosity mixture even through capillaries with small channel cross-section, transition to proportional section of $P_{L}=f(L / d)$ curve also proceeds without any pressure surge, as in the lower graph of Figure 4 [4].

Even though the non-invariant path of flow curves reflects the presence of nonstationary phenomena in the deformation zone, this did not exclude the possibility of application of experimental results for calculation of rheological indices of electrode coating mixtures. Derived results were used in analysis of possible causes for emergence of the above phenomena.

On total pressure losses. At total pressure losses $P_{L}=P_{0}+P_{c}$ its components $P_{0}$ and $P_{c}$ are not only summated, but also significantly influence $P_{L}$ value, due to their mutual influence. Mutual influence of complex function arguments is usually taken into account as their product, i.e. $\dot{\gamma}_{R} \dot{\gamma}_{\mathrm{sh}}$. Proceeding from dimensional considerations, their geometrical mean is used (in our case, $\omega=\sqrt{\dot{\gamma}_{R} \dot{\gamma}_{\text {sh }}}$ ).

If $\dot{\gamma}_{R}=2 V_{R} / D_{R}, \dot{\gamma}_{\mathrm{sh}}=2 V_{R} / d_{\mathrm{c}}\left(D_{R} / d_{\mathrm{c}}\right)^{2}$, then their product can be presented in the following form: $2 V_{R} / D_{R d c}\left(D_{R} / d_{\mathrm{c}}\right)^{2}=4 \beta^{2} V^{2} / D_{R} d_{c^{\circ}}$. In logarithmic modification the expression for geometrical mean has the following form: $\lg \omega=\lg \sqrt{4 \beta^{2} V_{R}^{2} / D_{R} d_{c}}$.

It is seen that pressure losses for forming the pressure flow of coating mixture are a complex function of the speed of the plunger, injecting the mixture into the flow zone, container and capillary diameters, as well as their ratio determining the degree of capillary jet reduction. Under non-isothermal conditions the influence of viscous heating of the mixture makes an additional contribution, which is concentrated in the zone of the highest gradient of the shear rate.

Calculations showed that in our experiments, $\dot{\gamma}_{R}$ fraction in the product of $\dot{\gamma}_{R} \dot{\gamma}_{\mathrm{sh}}$ varied in the range from 0.03 up to $21.5 \%$.

When $D_{R} \geq 30 \mathrm{~mm}$, the gradient of shear rate in the container is much smaller than that in the capillary, and its influence on $P_{L}$ can be neglected. At $D_{R} \leq$ $\leq 10 \mathrm{~mm}$ the influence of shear rate gradient becomes quite noticeable, and it should be taken into account.

Figure 5 shows $P_{L}$ dependence on $\omega$ in the pressure flow of S2 and N2 mixtures. In Figure 5, $a$ (S2 mixture) three sections can be singled out. Left and right
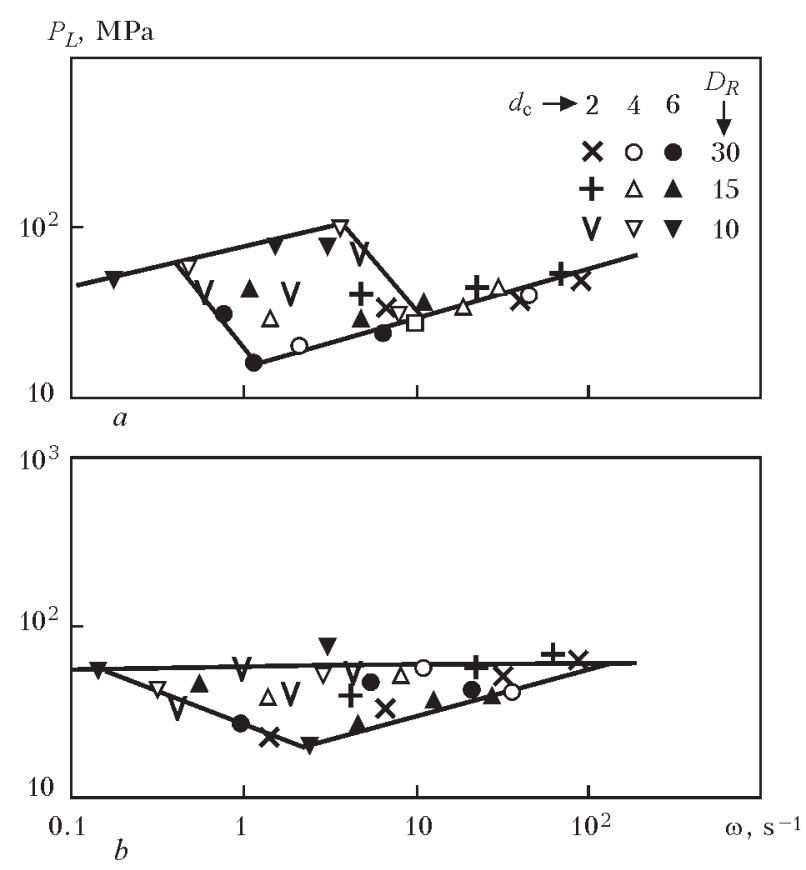

Figure 5. Dependence of total pressure losses in entrance zone and in capillary on $\omega$ value for S2 (a) and N2 (b) mixture

rectilinear sections are slow (creep) and fast steadystate flow, respectively. They are invariant to complex index of deformation mode. Located between them is the instability zone, resembling «stick-slip» flow mode. This kind of instability is often encountered in the technology of processing polymer materials and it is presented in the form of $\tau=f\left(\dot{\gamma}_{\mathrm{sh}}\right)$ or $\dot{\gamma}_{\mathrm{sh}}=f(\tau)$ graphs [9], and in one of the first publications [10] devoted to it - in the form of $s=f(\tau)$, where $s$ is the elastic, i.e. recoverable, strain.

It is believed that flow instability, recorded in the form of flow mode transition from the upper to lower, less energy-intensive flow mode, is associated with competition of the elastic and viscous forces.

In Figure 5, $b$ (N2 mixture) a rheogram of similar meaning has the form of a very narrow triangle, reflecting a noticeable scatter of results only in the case of application of small mixture containers. At $D_{R}$ increase up to $15 \mathrm{~mm}$ scatter is reduced, and when $D_{R}=30 \mathrm{~mm}$ no scatter is found.

$P_{0}$ dependencies on $\omega$ have similar, but not as pronounced form.

In Figure 6 the results, obtained when studying S2 mixture, are represented by $Q=f\left(P_{L}\right)$ dependence, characterizing the process of electrode manufacturing in the extruding machines with hydraulic drive, without $Q$ and $P$ recalculation to consistent variables $\tau$ and $\dot{\gamma}_{\text {sh }}$. It is seen that in such an interpretation they are quite similar to pressure-flow characteristic, described in [1].

It can be explained as follows. At low pressures (at $Q<1 \mathrm{~cm}^{3} \cdot \mathrm{s}^{-1}$ ) the coating is in the state of creep flow, in which the rate of its coagulation structure destruction 


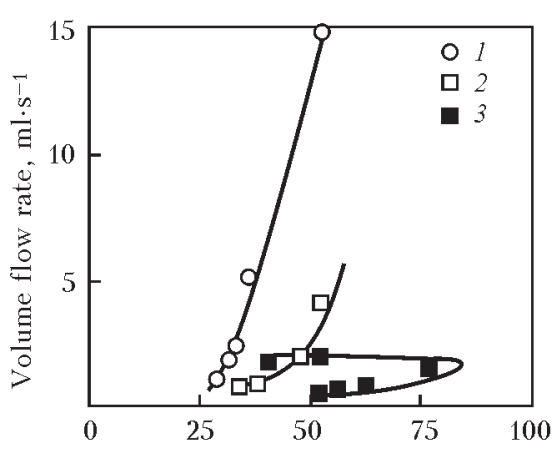

$a$

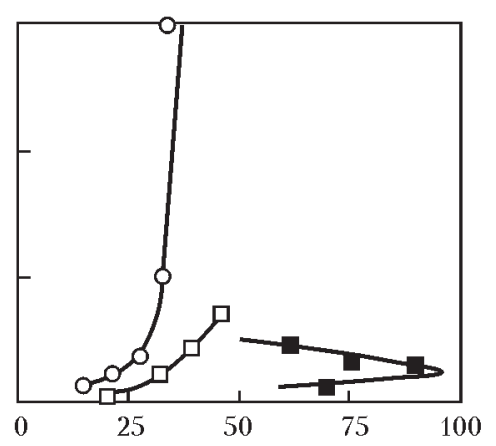

$b$

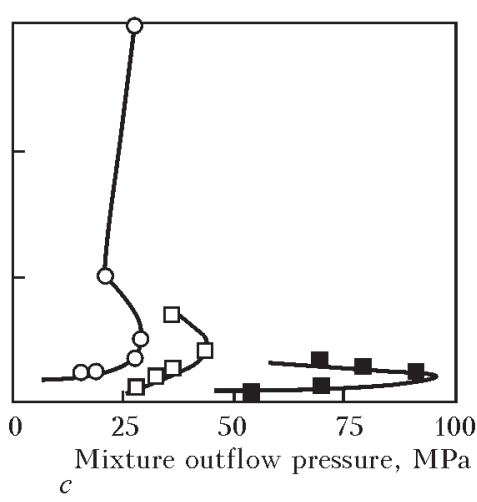

$c$

Figure 6. Pressure of mixture outflow from the container through capillary with channel diameter of $2(a), 4(b), 6(c) m m$ and $L=10 d_{c}$ at $D_{R}=30$ (1), 15 (2) and 10 (3) $\mathrm{mm}$

is close to the value of elastic stress relaxation rate. The role of mixture self-heating at this flow stage is insignificant. The flow rate rises with $P_{L}$ increase, and ever greater part of elastic stresses, not having enough time to relax, accumulates in the jet. After achievement of $Q=1 \mathrm{~cm}^{3} \cdot \mathrm{s}^{-1}$ the quantity of accumulated elastic energy is sufficient for flow stalling, which results in mixture structure breaking-up, its viscosity abruptly lowering, $P_{L}$ value decreasing, and further acceleration of the jet proceeds at smaller pressure built-up. No doubt that it is exactly the jet temperature that plays an important role in facilitating the jet stalling under the influence of elastic stresses and after it. It, similar to the rate, promotes destruction of the structure and lowering of mixture viscosity in the flow. Elasticity indices only slightly change here. Viscosity decreases to a much greater extent, together with the mixture ability to smooth the adverse influence of mixture elasticity on pressure flow stability.

Results presented in Figure 5 were obtained at application of constant flow viscosimeter with steplike flow regulation and discrete increase of flow rate by variation of jet cross-section. As a result, temperature and rate conditions of the flow changed in a step-like manner from experiment to experiment, resulting in pressure-flow curves also taking an intermittent shape.

Influence of the method of mode regulation on flow curves. The mixture flow in pre-capillary zone is deformed by shear and tension. Shear deformation in the tank before the capillary $\dot{\gamma}_{R}$ can be presented by expression $\dot{\gamma}_{R}=\dot{\gamma}_{\mathrm{sh}} / \beta^{3}$, where $\dot{\gamma}_{\mathrm{sh}}$ is the gradient of shear rate in the capillary, and $\beta=D_{R} / d_{\mathrm{c}}$ is the degree of jet reduction. Stretching of mixture flow, achieved during reduction and called Hencky strain, is presented as [11]:

$$
\varepsilon \equiv \int_{0}^{+\infty} \dot{\varepsilon} d t=\int_{v_{z}(-\infty)}^{v_{x}(+\infty)} \frac{d v_{z}}{v_{z}}=\ln \left(\beta^{2}\right),
$$

where $\dot{\varepsilon}$ is the tensile deformation rate.
In its turn, $\dot{\gamma}_{\mathrm{sh}}$ is related to Deborah number (or, which is the same, to elastic turbulence criterion $R e_{\mathrm{e}}$ ) by expression De $=\theta \dot{\gamma}_{\text {sh }}$.

We can see that the gradient of shear rate in the capillary and total extent of flow stretching are functions of one and the same argument $\beta$. Nonetheless, they react differently to its change. At one and the same $\beta$ value, $\dot{\gamma}_{R}$ increase should promote greater shear destruction of the mixture structure in the capillary, whereas with $\beta$ increase at $\mathrm{De}=$ const, greater stretching of the flow should be expected.

This follows from the results of work [11], in which $\beta$ and De influence on pressure flow structure of the so-called Boger fluid, was studied. Its composition, as a mixture of three polymer solutions, features specific rheological properties, namely, its elasticity index $\xi$ decreases essentially with increase of shear deformation rate, whereas shear viscosity $\eta$ remains almost unchanged. This may be the reason, why an almost complete range of instabilities, encountered in industrial processing of polymers, namely cyclic, quasiperiodic and vortex-like flow with formation of incoming, pulsed or rotating vortex, was found when studying Boger fluid in the region of comparatively small changes of $\beta$ and De.

Experiments were conducted with application of pneumatic capillary viscosimeter. To avoid undesirable vibrational impact of viscosimeter on investigation results, the controlled liquid was fed from the mixture tank to viscosimeter measuring cell through regulating valves, solely under the influence of static pressure. Circulation pump did not operate during measurements. To maintain the correct proportion of capillary and tank cross-sections, i.e. $\beta$, respective inserts were placed into the standard tank. The region of used flow modes was limited by Deborah number values in the range from 2 to 5 , and that of $\beta$ index in the range from 2 to 6 . Laser Doppler speedometer was used for jet speed measurement. Flow profile was recorded onto videotape by speed high-resolution TV 
camera. Experimental results are given in Figure 7. It shows the zones of instabilities revealed during the experiments.

As follows from the given graph, stable flow in the tank pre-capillary space is achieved in zone 1 at relatively low De values, and irrespective of Hencky strain magnitude used to create the flow.

Boundary of stability zone is limited by curve 2 . Its shape is indicative of the fact that at $\beta<4$, flow instability is in place already at quite limited increase of Deborah number. Contrarily, if $\beta>4$, flow modes can be used, which are characterized by ever higher De values, which are the higher, the greater $\beta$.

In our experiments, the range of $\beta$ value variation is by an order of magnitude higher, and that of De is by 2 orders higher than in study [11]. Nonetheless, as follows from Figure 5, dimensions of instability zone are also quite limited, particularly for S2 mixture.

Let us assume that the regularities revealed when studying Boger fluid as a model of viscoelastic material can be transferred to electrode coating mixtures. Then, judging by the graph in Figure 7, the only method to go around the instability zone at extrusion of electrode coating mixtures is applying flow forming mode with high values of the degree of jet compression, i.e. working with tanks of large enough diameter.

At very high values of $\beta$, providing sufficient distance from the right, steeply ascending branch of boundary 2, it will, apparently, be possible to increase the mixture deformation rate by the method of its ever faster injection by the plunger into the flow zone, without the risk of crossing the above boundary. For S2 mixture, this distance will be smaller than that for $\mathrm{N} 2$ mixture, in which this zone is of a much greater extent.

The validity of this assumption was verified by conducting experiments with $\mathrm{S} 3$ and $\mathrm{N} 3$ mixtures, extruded through holes in the locking disc of 2, 4 and $6 \mathrm{~mm}$ diameter from tanks of 30 to $90 \mathrm{~mm}$ diameter.

To reliably eliminate the influence of mixture column friction in the container on pressure losses, so-called back pressing was used, which is applied for the same purpose in metal extrusion technology [7]. In this case, the required series of measurements was performed, when extruding one billet in modes with flows, gradually increasing from experiment to experiment. The total range of changing of shear rate gradient was from 0.1 to $10^{5} \mathrm{~s}^{-1}$. Figure 8 gives the results.

It is seen that for high-viscosity $\mathrm{S} 3$ mixture the results are invariant in the entire range of $\dot{\gamma}_{\text {sh }}$ measurement.

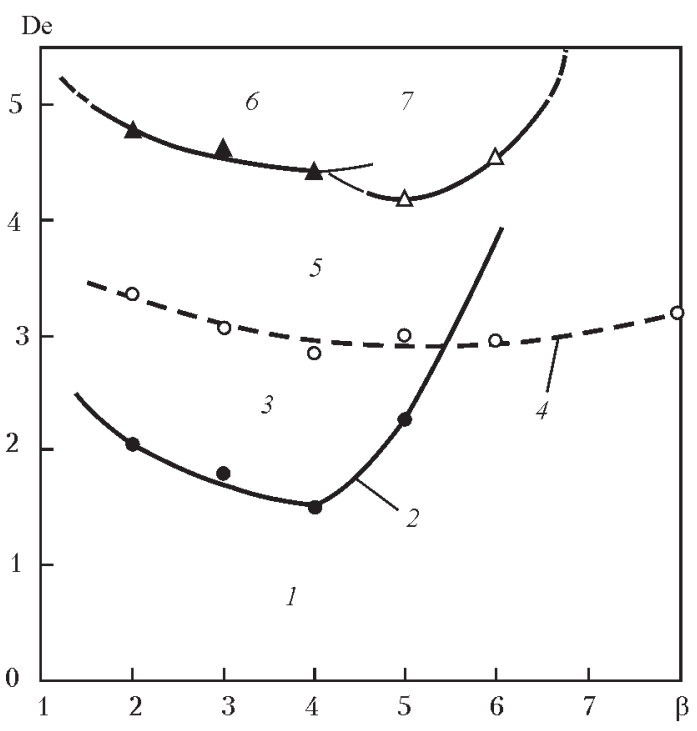

Figure 7. Kinds of flow instabilities in axially symmetric abruptly narrowing channel depending on ratio of the degree of flow contraction and elastic turbulence criterion: 1 - steady flow; 2 , 4 - boundaries of steady and vortex-like flow; 3, 5, 6- cyclic, quasiperiodic, pulsed flow, respectively; 7 - rotating vortex [11] Therefore, at $D_{R} \geq 30 \mathrm{~mm}$ its pressure flows are not prone to instability in a very broad range $\left(\dot{\gamma}_{R}\right)$.

Low-viscosity N3 mixture is prone to flow instability, caused by unrelaxed elastic stresses. Invariance of its $P_{0}=f\left(\dot{\gamma}_{\text {sh }}\right)$ flow curves is in place until $D_{R}$ has reached $70 \mathrm{~mm}$. The higher $D_{R}$, the smaller is the section of flow curve non-invariance. At $D_{R}=70 \mathrm{~mm}$ the non-invariance disappears.

The higher $D_{R}$, the smaller the flow index $n_{0}$, that is indicative of increase of the degree of Newtonian behaviour of the coating mixtures, and, therefore, of the increase of coating fluidity, as a result of greater destruction of their structure and resulting smaller accumulation of elastic energy in the flow. The greater the container cross-section, the lower is the flow rate, at which the curve reaches the saturation section. In S3 mixture the curves were eventually completely flattened.

Based on the data, which we have, the influence of initial viscosity of extruded material on jet temperature gradually decreases with increase of the degree of pressing, and at sufficiently high $\beta$ values it becomes insignificant.

By the results of studying S3 mixtures, values of extrusion forces and pressures $P_{0}$ were calculated after the flow curve has reached the saturation section. Calculation results are given in Table 3. They show that at increase of container cross-section area 3.2 times, the pressing force increased just 2.2 to 2.8 times. Pressing pressure decreased to the greatest extent at maximum value of jet compression: 1.2-2.1 times at hole diameter $d_{\mathrm{C} 0}=2 \mathrm{~mm}$. The greater $d_{\mathrm{C} 0}$, the weaker is the influence of $\beta$ parameter on $P_{\mathrm{C} 0}$ 

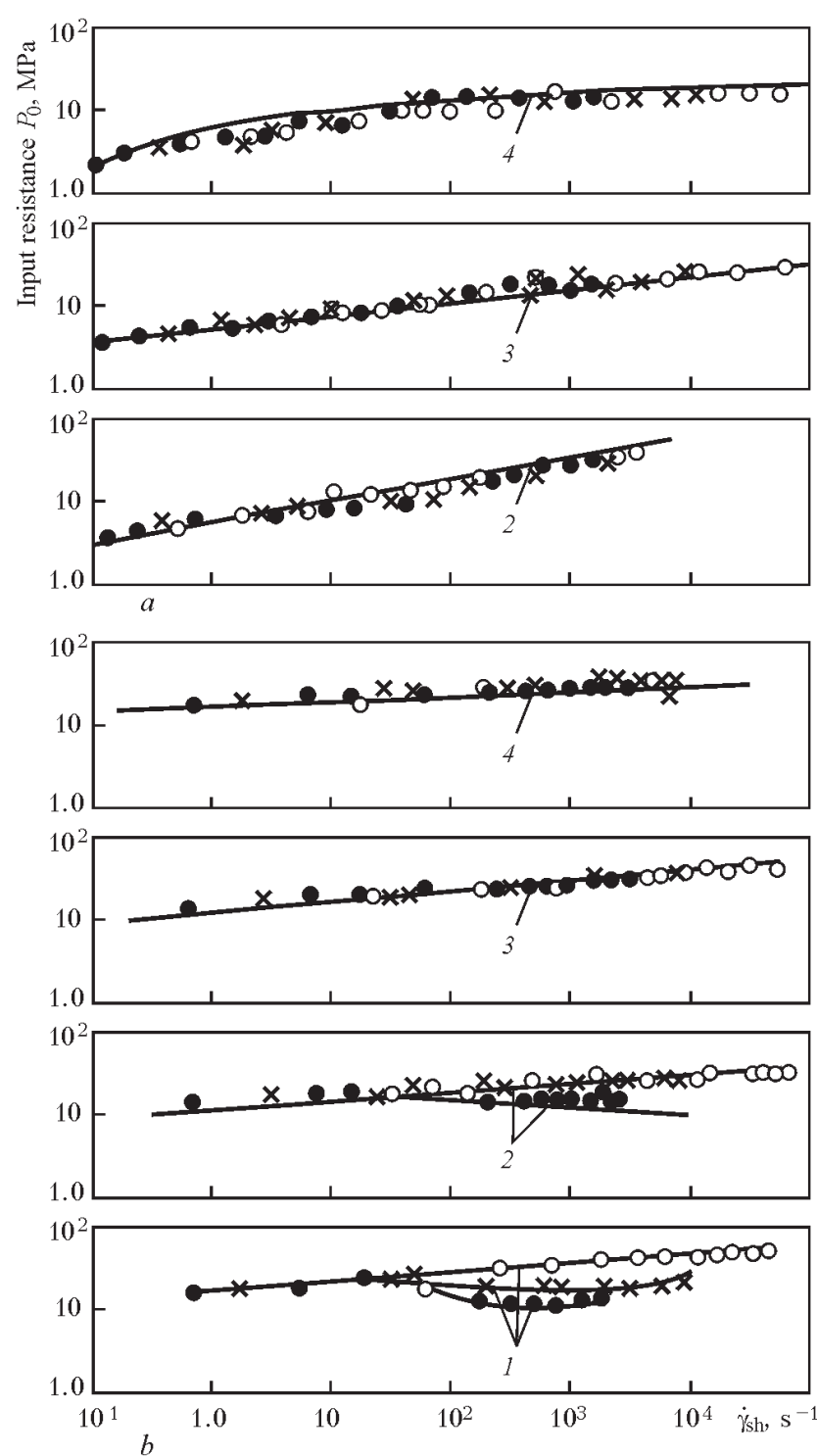

Figure 8. Pressure-flow characteristics of coating mixtures S2 (a) and N3 $(b)$ obtained with application of mixture containers with $D_{R}=$ $=50$ (1), 70 (2), 90 (3) (a) and 30 (1), 50 (2), 70 (3), 90 (4) $\mathrm{mm}(\mathrm{b})$

Characteristics of viscoelasticity of the compared coating mixtures. Figure 9 gives the dependence of S2 and N2 mixture relaxation period on shear rate gradient, and Figure 10 - the influence of shear rate gradient on shear viscosity and normal stress coefficients, as well as influence of average tensile deformation rate on longitudinal viscosity of $\mathrm{S} 2$ and $\mathrm{N} 2$ mixtures.

As follows from Figure 9, the compared mixtures are little different from each other by the influence of deformation rate on characteristic time (relaxation period $\theta$ ). Values of $\dot{\gamma}_{\mathrm{sh}}$, at which functions $\theta\left(\dot{\gamma}_{\mathrm{sh}}\right)$ cross horizontal $\theta=1$, are approximately the same $\left(\dot{\gamma}_{\mathrm{sh}} \approx 40-60 \mathrm{~s}^{-1}\right)$ for the compared coatings. However, scattering of experimentally found $\theta$ values is greater in N2 mixture than in S2 mixture. So, in S2 mixture ratio $\theta=\eta / G$ was close to 1 only in two experiments $\left(\beta=2.5, \dot{\gamma}_{\mathrm{sh}}=50 \mathrm{~s}^{-1}\right.$ and $\left.\beta=2.5, \dot{\gamma}_{\mathrm{sh}}=35 \mathrm{~s}^{-1}\right)$.
Table 3. Mixture extrusion characteristics

\begin{tabular}{|c|c|c|c|}
\hline \multirow{2}{*}{$D_{R}, \mathrm{~mm}$} & $d_{\mathrm{Co}}, \mathrm{mm}$ & Pressing force, $\mathrm{t}$ & $P_{0}, \mathrm{MPa}$ \\
\hline \multirow{3}{*}{50} & 2 & 47.8 & 0.330 \\
\cline { 2 - 4 } & 4 & 45.1 & 0.230 \\
\cline { 2 - 4 } & 6 & 34.3 & 0.180 \\
\hline \multirow{3}{*}{70} & 2 & 98.2 & 0.255 \\
\cline { 2 - 4 } & 4 & 84.7 & 0.220 \\
\cline { 2 - 4 } & 6 & 69.3 & 0.180 \\
\hline \multirow{3}{*}{90} & 2 & 101.7 & 0.160 \\
\cline { 2 - 4 } & 4 & 98.6 & 0.155 \\
\cline { 2 - 4 } & 6 & 95.4 & 0.155 \\
\hline
\end{tabular}

Testing low-viscosity mixture revealed five such cases $\left(\beta=7.5, \dot{\gamma}_{\text {sh }}=40 ; \beta=3.75, \dot{\gamma}_{\text {sh }}=40, \beta=7.5\right.$, $\dot{\gamma}_{\mathrm{sh}}=40 ; \beta=5, \dot{\gamma}_{\mathrm{sh}}=50$; and $\beta=2.5, \dot{\gamma}_{\mathrm{sh}}=22 \mathrm{~s}^{-1}$ ).

Works $[2,3]$ showed that $\theta=\eta / G=1$ is a necessary, but not always sufficient condition for formation of positive pulsed modes of mixture flow through the capillary. Whether they actually originate in the system of the tank and capillary, and whether they are in place for a long time after that, will be determined by ratio $\eta / \xi$, which is reached at moment $\theta=1$. As follows from Figure 10, mixture structure destruction results in $\eta\left(\dot{\gamma}_{\mathrm{sh}}\right)$ and $\xi\left(\dot{\gamma}_{\mathrm{sh}}\right)$ functions decaying with $\dot{\gamma}_{\text {sh }}$ increase, the second one more steeply than the first one, so that they cross each other. To the left of crossing point $\xi>\eta$, so that mixture viscosity is not capable of damping the influence of elastic stresses. Such ratio will be in place right down to zero flow rates, as $\xi_{0}=\left(\eta_{0}\right) \theta$ [12]. To the right of the crossing point $\xi<\eta$, so that such an ability of the mixtures is the greater, the higher $\dot{\gamma}_{\mathrm{sh}}$.

It also follows from Figure 10 that relative position of $\eta\left(\dot{\gamma}_{\text {sh }}\right)$ and $\xi\left(\dot{\gamma}_{\text {sh }}\right)$ straight lines is not the same, because of lower viscosity of N2 mixture. Therefore, the point of crossing of competing functions in S2 mixtures lies much more to the left than does $\dot{\gamma}_{\mathrm{sh}}$ critical value (designated by vertical arrow), at which condition $\theta=1$ is fulfilled. Consequently, there is sufficient margin of $\eta\left(\dot{\gamma}_{\mathrm{sh}}\right)$ excess over $\xi\left(\dot{\gamma}_{\mathrm{sh}}\right)$, so as to counteract initiation of pressure flow pulsed mode.

In N2 mixture the point of crossing of compared functions $\eta\left(\dot{\gamma}_{\mathrm{sh}}\right)$ and $\xi\left(\dot{\gamma}_{\mathrm{sh}}\right)$ also lies to the left of $\dot{\gamma}_{\text {sh }}$ critical value, but much closer to it. As a result, excess of damping function $\eta\left(\dot{\gamma}_{\mathrm{sh}}\right)$ over $\xi\left(\dot{\gamma}_{\mathrm{sh}}\right)$ function, capable of causing elastic disturbances, can be regarded to be sufficient to prevent the possibility of pulsed flow initiation, but not always enough for completely suppressing such a possibility. This is confirmed by the shape of extrusion curves, partial relaxations of elastic stresses in the form of pressure relieving, as well as essential scattering of results. 

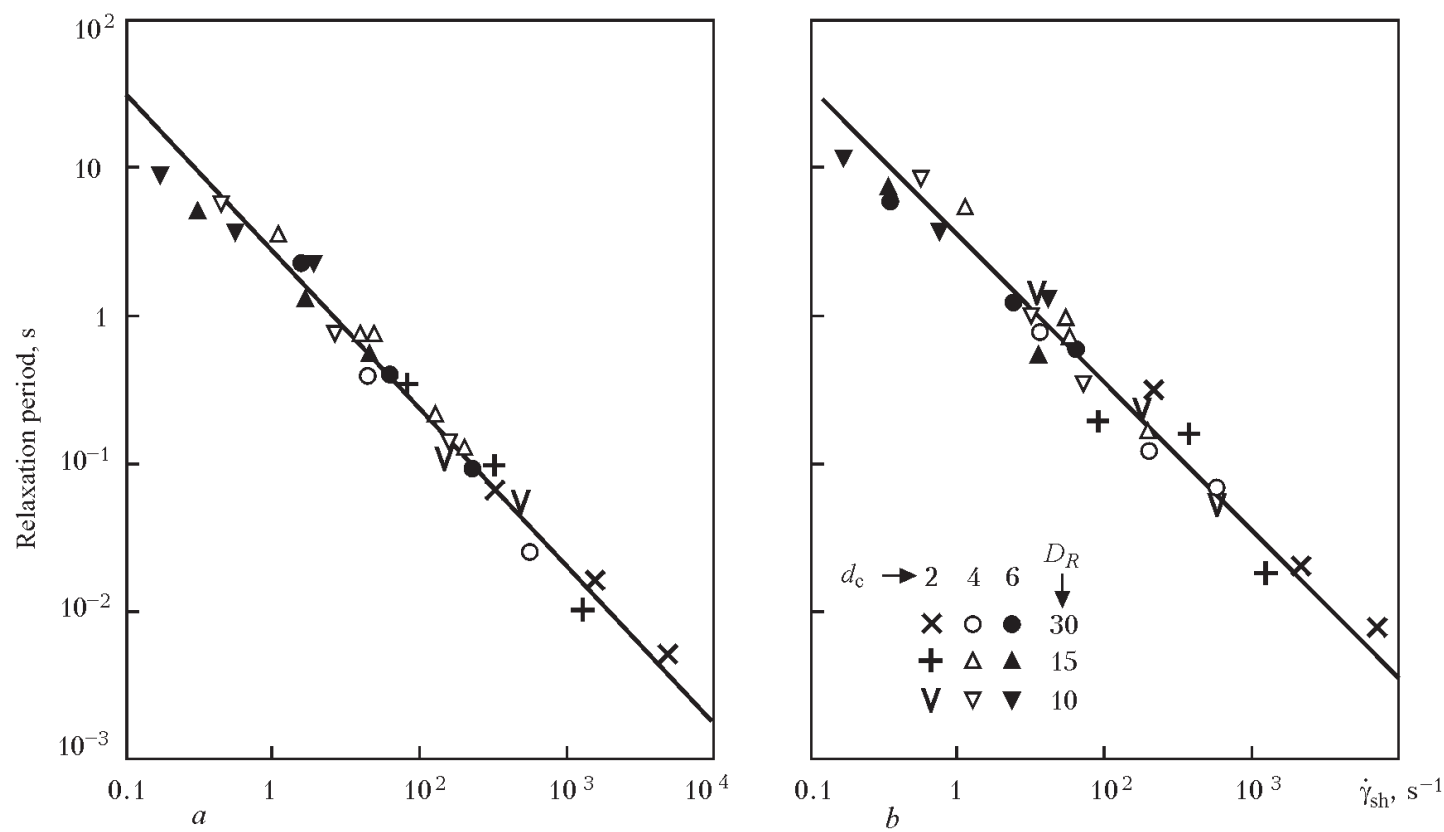

Figure 9. Dependence of relaxation period of coating mixtures S2 (a) and N2 (b), extruded from tanks with $D_{R}=10,15$ and $30 \mathrm{~mm}$ through capillaries with $d_{c}=2,4$ and $6 \mathrm{~mm}$, on shear rate gradient

Accumulation of elastic stresses is accompanied by reduction of the angle of natural convergence of flow $\alpha_{0}$. Figure 11 shows the influence of the criterion of elastic turbulence in flows of S2 and $\mathrm{N} 2$ mixtures on $\alpha_{0}$ value. It is seen that viscous mixture is characterized by much smaller scatter of elastic turbulence characteristic and associated $\alpha_{0}$ value, compared to the low-viscosity mixture. This is confirmed by statistical estimate of the obtained results given in Figure 12.

Thus, it is anticipated that approaches applied for achievement of similar purposes in the technology of polymer material extrusion can be beneficial in terms of increasing flow stability of mixtures, designed for coating welding electrodes. They are based on the concepts of existence of an inextricable
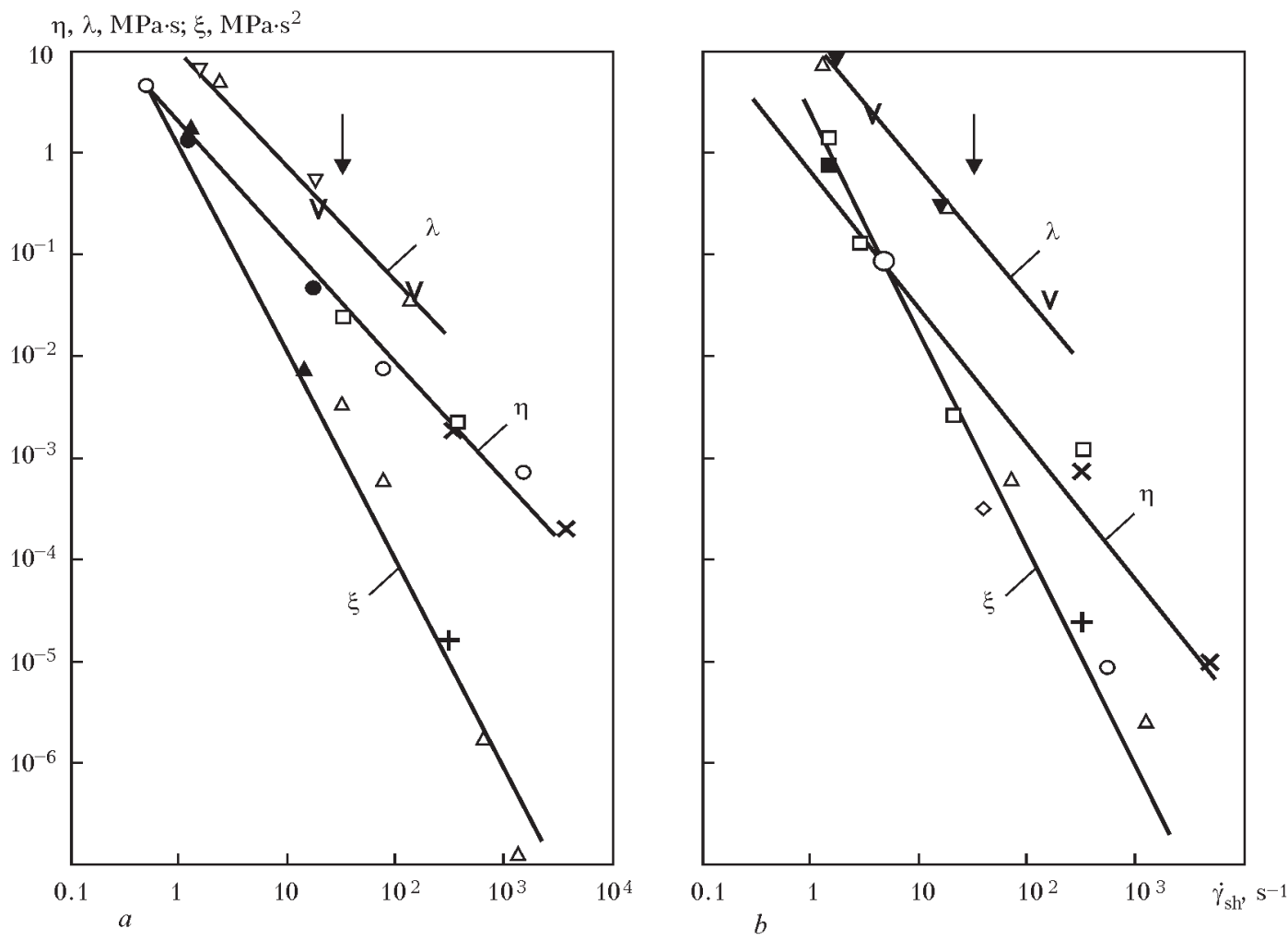

Figure 10. Dependence of $\eta, \xi$ and $\lambda$ on shear and longitudinal flow for S2 (a) and N2 (b) mixture (arrows indicate $\dot{\gamma}_{\text {sh }}$ value, at which condition $\theta=1$ is fulfilled) 

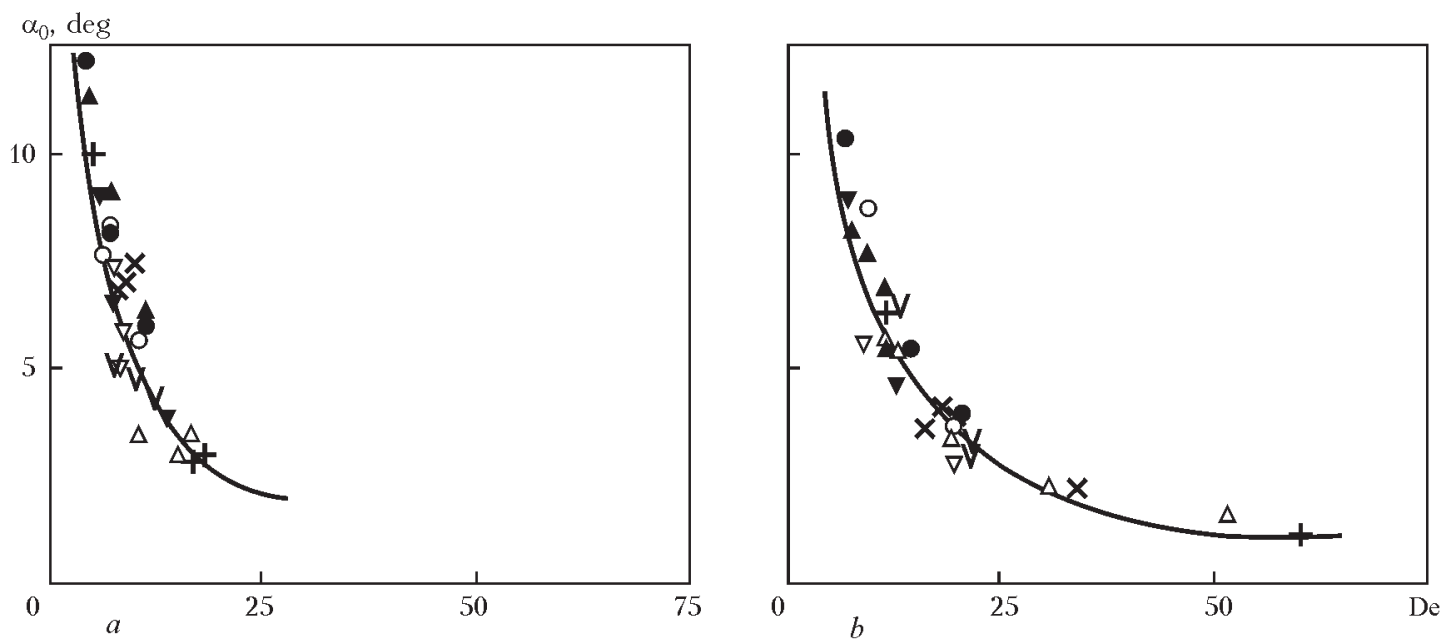

Figure 11. Influence of Deborah number on angle of natural convergence of coatings mixtures S2 $(a)$ and N2 (b) extruded from tanks with $D_{R}=10,15$ and $30 \mathrm{~mm}$ through capillaries with $d_{\mathrm{c}}=2,4$ and $6 \mathrm{~mm}$

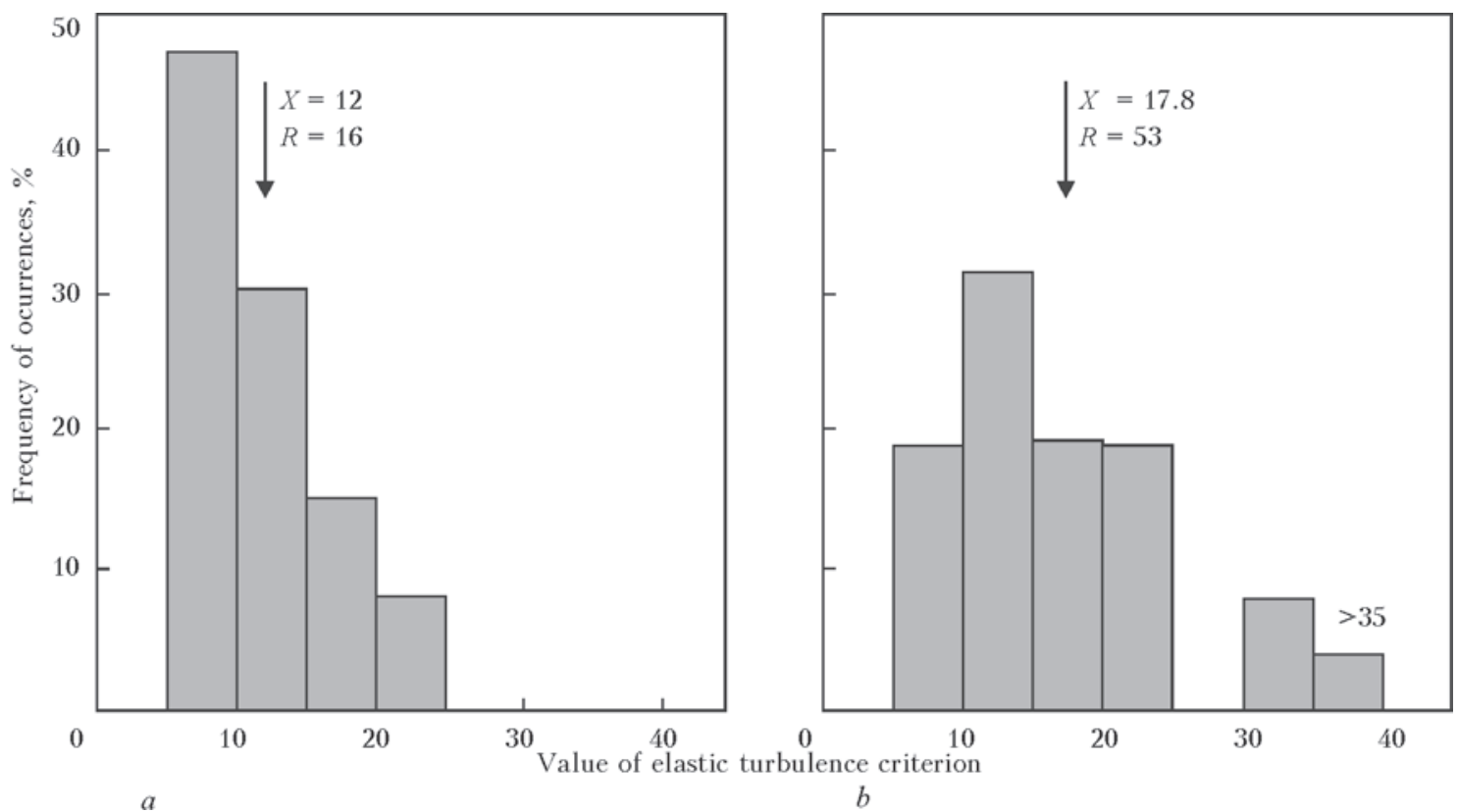

Figure 12. Statistical distribution of Deborah number as characteristic of angle of natural convergence of pressure flows of coating mixtures S2 $(a)$ and N2 $(b)$ extruded from tanks with $D_{R}=10,15$ and $30 \mathrm{~mm}$ through capillaries with $d_{\mathrm{c}}=2,4$ and $6 \mathrm{~mm}: X-$ average sample value; $R$ - sampling scope

link between the two effects, specific for polymer systems, namely appearance of the so-called viscosity anomaly at flowing with increase of deformation rate, on the one hand, and generation of normal stresses, caused by accumulation of recoverable strains, on the other. Normal stresses in a certain range of rather slow deformations are capable of provoking various kinds of flow instabilities. Their appearance can be prevented, if normal stresses are damped by system viscosity. Such a possibility exists due to the fact that effective viscosity decreases slower with increase of deformation rate than does the coefficient of normal stresses [12].

\section{Conclusions}

1. Model of continuous-flow stepped channel simulating the conditions of electrode coating mixture flow in measuring cell of capillary viscosimeter or forming head of electrode extrusion machine was used to study the structure and rheological characteristics of non-isothermal mixture flow for low-hydrogen electrodes. Mixtures, one with viscous, and another one with low-viscosity liquid glass, differed significantly by the degree of structuredness, viscosity and elasticity ratio and resistance to instability appearance in pressure flows.

2. It is established that the compared mixtures react differently to capillary testing modes, which were adjusted by changing the flow rate or degree of jet reduction. Mixture with low-viscosity glass is more structured and, therefore, it is less prone to dissipative heating than its viscous analog, as part of the energy spent for flowing is consumed by structure 
destruction. On the other hand, the above-mentioned methods of flow mode regulation are inadequate to each other, either: speed increase by jet reduction causes its lower heating than does increase of mixture flow rate, irrespective of the method of reduction; be it by increase of tank cross-section or reduction of capillary cross-section.

3. Competition of elastic and viscous elements provokes all kinds of irregularities in coating mixture pressure flows. They are revealed by visualization (as a change of flow pattern), on recorded extrusion curves (as flow irregularities and stalling), on flow curves (as different degree of their non-invariance) and as a change of total pressure losses, caused by elastic stress relaxation. Here, elasticity can be regarded as a provoking, and viscosity - as a damping factor. Viscous heating increases the probability of instability development, as, similar to flow rate, it lowers mixture viscosity, while breaking up its structure.

4. Tank and capillary are the sites of instability development. In the work, it is proposed to allow for the role of each stage in appearance, for instance, of instability of total pressure losses in the tank and the capillary, in the form of the function of complex argument $\omega$ as geometrical mean of gradients of shear rate in the tank and in the capillary. Such an approach allowed establishing the critical range of pressure flow modes, within which function $P_{L}(\omega)$ is exclusively non-invariant. Beyond this range $P_{L}(\omega)$ flow curves have an invariant form.

5. Ratio of viscous and elastic properties in the compared mixtures should be evaluated by comparing shear viscosity $\eta$ with modulus of elasticity $G$, on the one hand, and shear viscosity with the coefficient of first difference of elastic stresses $\xi$, on the other hand. Equality of $\eta$ and $G$ characteristics in the first ratio is the mandatory condition for appearance of disturbances in the capillary flow. Whether they actually arise and will be prolonged, is determined from the value of $\eta / \xi$ ratio at moment of time, when $\eta=G$. If $\eta>\xi$, the disturbances, even the arisen ones, for instance, in the form of flow pulsations, cannot be prolonged as dynamic viscosity damps elasticity, represented by coefficient $\xi$ of similar meaning. Values of coefficients $\eta$ and $\xi$ decrease with increase of shear rate gradient, steeper for the second of them than for the first one. In the graphic form $\eta\left(\dot{\gamma}_{\mathrm{sh}}\right)$ and $\xi\left(\dot{\gamma}_{\mathrm{sh}}\right)$ functions cross. To the right of the crossing point, condition $\eta>\xi$ is fulfilled to the greater extent, the higher $\dot{\gamma}_{\mathrm{sh}}$, and even when $\eta=G$, the arisen disturbance will be short-term, or will be implemented in the form of individual jet stalling. To the left of crossing point of $\eta\left(\dot{\gamma}_{\text {sh }}\right)$ and $\xi\left(\dot{\gamma}_{\text {sh }}\right)$ functions, condition $\eta<\xi$ is satisfied, and this opens up broad possibilities for emergence of prolonged disturbances.

6. Kinds and, particularly, intensity of disturbances in pressure flows of coating mixtures depend on the method of their speed regulation: by jet reducing $\beta$ or by the rate of mixture injection into zone of reduction by the plunger. Increase of $\beta$, owing to large tank diameters together with smaller heating of the mixture, weakens the influence of peripheral elastic zones on pre-capillary space, in which the jet forms, lowers the level of stored elastic energy and, therefore, the flow curve invariance. Moreover, the share of material deformation by tension is increased that facilitates maintaining the flow stability. The larger the tank diameter, the lower the pressure required for ensuring a stable pressure flow of coating mixtures.

7. Coating mixtures based on low-viscosity liquid glass are more prone to emergence of disturbances in pressure flows than their viscous analogs.

1. Sokolov, E.V. (1950) Electrodes with quality coating and their production. Avtogen. Delo, 11, 26-29.

2. Marchenko, A.E. (2014) Thickness difference of electrode coating caused by elastic turbulence of electrode compounds under the conditions of nonisothermal pressure flow. The Paton Welding J., 6/7, 177-189.

3. Marchenko, A.E. (2014) Effect of charge grain composition on rheological characteristics of coating mixtures for lowhydrogen electrodes. Ibid., 163-171.

4. Marchenko, A.E. (2015) Examination of viscoelastic behavior of electrode coating mixtures in the state of pressure flow through cylindrical forming orifices. In: Proc. of St.Petersburg Int. Sci.-Techn. Conf. on Welding Consumables (15-17 Oct. 2015), 79-89.

5. Marchenko, A.E., Gnatenko, M.F. (1980) Specifics of flow of electrode coating mixtures, detected by capillary plastometer: Inform. Doc. of SMEA, Issue 1, 106-117.

6. Marchenko, A.E. (2015) About thermal conditions, generated by viscous heating of electrode coating mixture in the zone of pressure flow formation. In: Proc. of St.-Petersburg Int. Sci.Techn. Conf. on Welding Consumables (15-17 Oct. 2015), 65-78.

7. Perlin, I.L. (1964) Theory of pressing of metals. Moscow: Metallurgiya.

8. Yankov, V.I., Glot, I.O., Trufanova, N.M. et al. (2010) Flow of polymers in die holes. Theory, calculation, practice. Moscow, Izhevsk.

9. Georgios, G. (2005) Stick-slip instability. In: Polymer melts instabilities, control and understanding. N.Y.: Dekker, 176-221.

10. Philippoff, W., Gaskins, F.H. (1958) The capillary experiment in rheology. Transact. Soc. Rheol. II, 263-284.

11. McKinley, G.H., Raiford, W.P., Brown, R.A. et al. (1991) Nonlinear dynamics of viscoelastic flow in axisymmetric abrupt contractions. J. Fluid Mech., 223, 411-456.

12. Vinogradov, G.V., Malkin, A.Ya. (1977) Rheology of polymers. Moscow: Khimiya. 